\title{
Iranian Nephrology and Urology Research Output in the Past Two Decades: A Bibliographic Analysis of Medline Database
}

\author{
Behzad Einollahi ${ }^{1} ;$ Mohsen Motalebi $^{1, *} ;$ Mehrdad Taghipour $^{1} ;$ Mehrdad Ebrahimi $^{1}$ \\ ${ }^{1}$ Nephrology and Urology Research Center, Baqiyatallah University of Medical Sciences, Tehran, IR Iran \\ ${ }^{*}$ Corresponding author: Mohsen Motalebi, Nephrology and Urology Research Center, Baqiyatallah University of Medical Sciences, Tehran, IR Iran. Tel:+98-2181262073, Fax:+98-2181262073, \\ E-mail:dr.motalebi@ymail.com \\ Received: June 20, 2015; Accepted: July 17, 2015
}

\begin{abstract}
Context: We performed a bibliometric search to evaluate the number of papers published in the field of nephrology and urology by Iranian researchers in the past two decades.

Evidence Acquisition: We did an online search in abstract/title part of articles with 129 keywords such as kidney, renal, hemodialysis, transplant, nephrology, glomerulonephritis, ureteral, nephrolithiasis, and etc. Endnote software version 7 was used to search articles published in PubMed database from November 1993 to November 2013. Those articles in which Iran was the affiliation of at least one of the authors were selected. These articles in the field of nephrology and urology were analyzed regarding the name of originated institution, field of study, total number of publications, type of study, collaboration rate of Iranian nephrologist and urologists for every year, annual sharing of Iranian articles in five journals with highest impact factor (IF) and journal IF.

Results: The total number of publications in the field of nephrology and urology was 3,771 (average of 189 papers per year). Most of the Iranian nephrology and urology papers were from the capital city, Tehran (50.03\%). There was an increasing trend in the number of publications over the years. Most papers were about transplantation (44.6\%), nephrology (20.9\%) and hemodialysis (16.4\%). Of all, 53.7\% were retrospective articles, whereas the proportion of clinical trials was relatively small (10.8\%).

Conclusions: Although Iranian publications in the field of nephrology and urology have had a considerable and significant increase in the recent years amongst the Middle Eastern countries, there is a wide distance to be a science exporter country.
\end{abstract}

Keywords: Nephrology; Urology; Transplantation; Bibliographic

\section{Context}

Development of a community health depends on many different factors. Scientific studies play an important role among them (1). During the past two decades, Iran has enjoyed considerable expansion of trained individuals in the fields of medicine (2) for being a developed and industrial country. Therefore, the policy of Iranian governors changed in the recent years to provide a good condition for scientists and training researchers and increase science production. Iran has been successful in its agenda to improve its international profile in biomedical sciences by constantly increasing the quantity and quality of articles published in peer-reviewed biomedical journals indexed in PubMed. However, to date, little is known about contributions of Iranian medical researchers and their quality in the field of nephrology and urology. By examining various aspects of such investigations regarding quality and quantity of articles in the field of nephrology and urology, we would be able to reflect Iran's scientific progress and impact on world science production.
This is one of the earliest studies assessing Iran research productivity in the field of urology and nephrology. Findings of our study would provide a baseline data for assessment of future researches. Moreover, a precise and extensive view of the overall research productivity at regional and international level is presented here. The aim of this study was to evaluate the quality and quantity of Iranian papers in the field of nephrology and urology indexed in PubMed in the past two decades. This can help scientists and governors to compare this study with other counties and better future planning in nephrology and urology field.

\section{Evidence Acquisition}

\subsection{Keywords and Search Strategy}

We conducted a retrospective study to determine quality and quantity of nephrology and urology research output in the past two decade by Iranian scien-

Copyright ( ) 2015, Nephrology and Urology Research Center. This is an open-access article distributed under the terms of the Creative Commons Attribution-NonCommercial 4.0 International License (http://creativecommons.org/licenses/by-nc/4.0/) which permits copy and redistribute the material just in noncommercial usages, provided the original work is properly cited. 
tists. Two faculty members of Baqiyatallah University of Medical Sciences (one nephrologist and one urologist) helped us to find all relevant keywords in the field of nephrology and urology. We did an online search in abstract/title part of articles with 129 obtained keywords such as kidney, renal, hemodialysis, transplant, nephrology, glomerulonephritis, ureteral, nephrolithiasis, and etc. Endnote software version 7 (Bld 7072) was used searching PubMed database from November 1993 to November 2013. All articles in which Iran was the affiliation of at least one of the authors were selected. After deleting duplicate records, two investigators evaluated title and abstracts of searched articles and excluded papers unrelated to nephrology and urology. In case of disagreement between investigators regarding excluding or including an article, final decision was made by the professor of nephrology (B E). We gathered a total of 4064 relative articles as an offline database for further evaluation.

\subsection{Data Gathering}

The final offline database of Iranian articles in the field of nephrology and urology was analyzed as follows: 1) name of originated institution according to the first author address, 2) field of study, categorized as transplantation, hemodialysis, peritoneal dialysis, nephrology, urology, and other issues, 3) total number of publications, 4) Type of study (retrospective, prospective, clinical trial, case report and etc.), 5) collaboration rate of Iranian nephrologists and urologists for every year and the whole studied period, 6) annual publication of Iranian articles in five journals with highest impact factor (IF) regarding nephrology and urology, 6) name of journals publishing most Iranian papers, 7) articles published in journals with IF $\leq 1$, between 1 and 3 and more than 3 in each five years, and 8) the average of Iranian articles IF in each five years.

\subsection{Statistics}

All data were analyzed using SPSS Analytical Software version 19.0 (PASW, SPSS Inc., IMB Corporation, Chicago, Illinois, USA). Continuous variables were expressed as mean \pm standard deviation. Categorical variables were expressed as percentages. We used one-way ANOVA to compare categorized IF obtained in each year in case of normality; otherwise, Kruskal-Wallis-H test was used. P value of $<0.05$ was considered statistically significant.

\section{Results}

Our entire search results with 129 keywords were 27,503 articles. Deleting duplicate records yielded 8,602 papers. After evaluating title and abstracts by investigators, 3,771 relevant articles remained. An increasing trend in the number of publications from
1993 to 2013 was noted. Slightly more than $80 \%$ of papers published within seven recent years (2007 - 2013). Figure 1 shows the increase in the annual number of Iranian articles in the field of nephrology and urology in the past two decades.

The first five journals that published Iranian papers in the field of nephrology and urology were as follows: urology journal (9.7\%), Iranian journal of kidney disease (8.4\%), Saudi journal of kidney disease and transplantation (6.5\%), journal of endourology (1.9\%) and experimental and clinical transplantation (1.7\%). An overall of 1070 (28.3\%) Iranian articles were published in the mentioned five journals. Iranian journals published $59.8 \%$ and non-Iranian journals $40.2 \%$ of all papers. There was a significant difference between the mean IF of journals published in Iranian papers in different years $(P=0.03)$. Figure 2 shows the mean of journals IF in five years. We also categorized IF of journals to less than one, between one and three and more than three and then evaluated them in five years (1994, 1998, 2003, 2008 and 2013). Table 1 shows the percentage of IF categories in five years.

Transplantation (44.6\%), nephrology (20.9\%), hemodialysis (16.4\%), and infertility (11.8\%) were the most prevalent topics of papers. However, chronic ambulatory peritoneal dialysis (1.7\%) and other topics (4.6\%) contributed to a small proportion of publications. Figure 2 demonstrates the institutional affiliation of corresponding authors who published more than ten papers in the past two decades in nephrology and urology. Most Iranian nephrology and urology papers were from the capital city, Tehran (50.03\%), particularly from Tehran university of medical sciences (UMS; 18\%), Shahid Beheshti UMS (13.3\%), Baqiyatallah UMS (6.3\%), Iran UMS (3.1\%), Royan institute for reproductive biomedicine (1.8\%), Tarbiat Modares university (1.4\%), Avicenna research institute (0.83), Pasteur institute (0.4\%), and other institutes in Tehran (4.9\%). Of all papers evaluated in this study, 26.1\% were fundamental (Basic Science) and 53.7\% were retrospective articles. Clinical trials constituted $10.8 \%$ and case reports, $8 \%$ of the investigations.

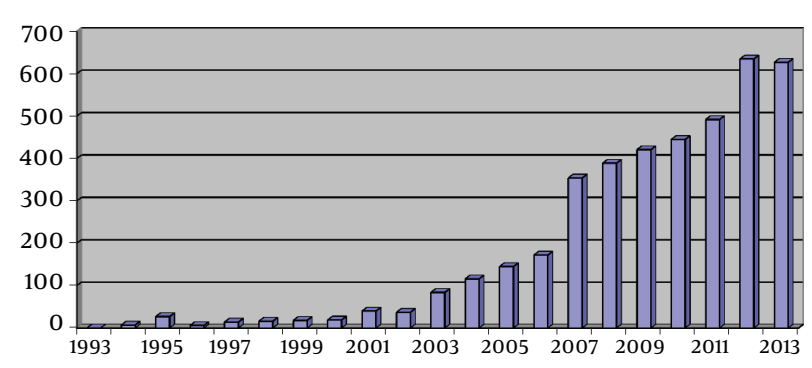

Figure 1. Increase in Annual Number of Iranian Papers Indexed in PubMed in the Field of Nephrology and Urology 


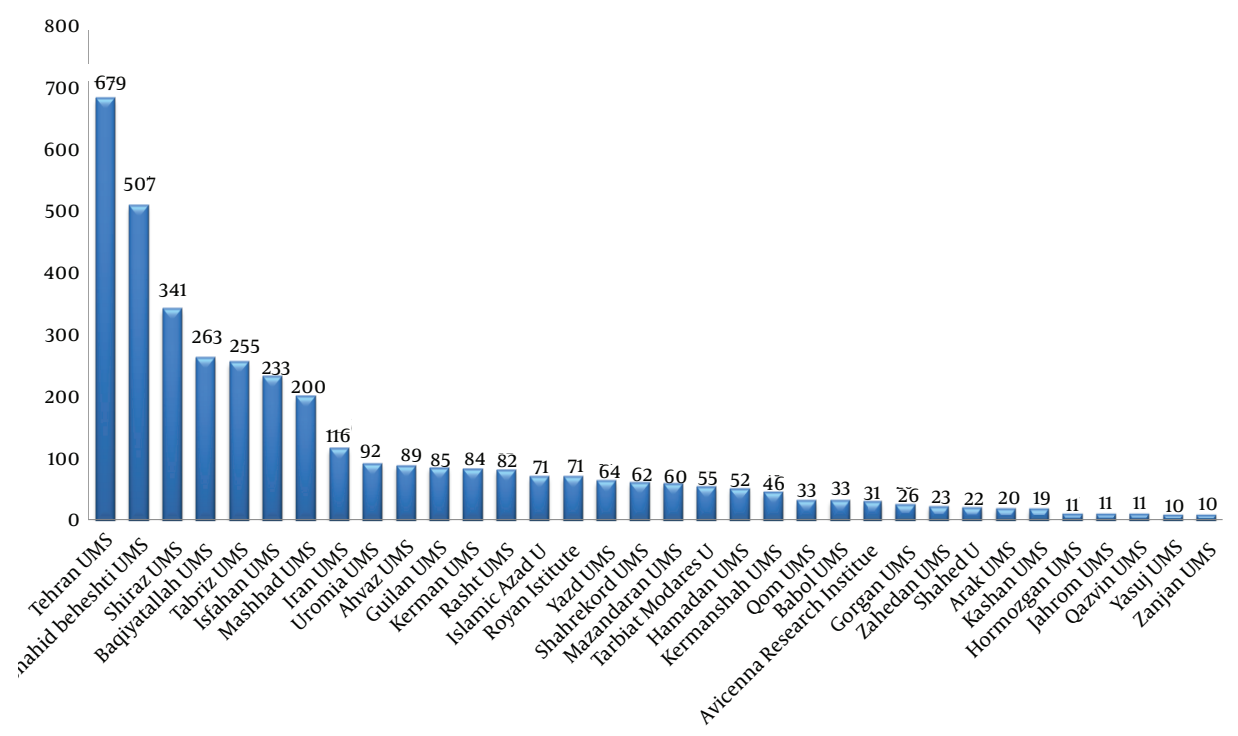

Figure 2. The Number of Nephrology and Urology Papers Published by Iranian Universities From 1993 to 2013

\begin{tabular}{|c|c|c|c|c|c|}
\hline IF $^{\mathbf{b}}$ & 1994 & 1998 & 2003 & 2008 & 2013 \\
\hline$\leq 1$ & $1(25)$ & $0(0)$ & $4(16)$ & $62(35.8)$ & $123(38.2)$ \\
\hline$>1,>3$ & $1(25)$ & $4(50)$ & $16(64)$ & $87(50.3)$ & $152(47.2)$ \\
\hline$\geq 3$ & $2(50)$ & $4(50)$ & $5(20)$ & $24(13.9)$ & $47(14.6)$ \\
\hline Total & $4(100)$ & $8(100)$ & $25(100)$ & $173(100)$ & $322(100)$ \\
\hline
\end{tabular}

${ }^{\mathrm{a}}$ The values are presented as No. (\%)

b Abbreviation: IF: impact factor.

\section{Conclusions}

Iranian publications in the field of nephrology and urology have been increased during the past two decades. As shown in Figure 1, the number of Iranian indexed publications in PubMed had a considerable increase since 2006; publications number increased from less than 200 publications in 2006 to more than 300 publications in 2007. Since that year, Iranian researchers have had a significant growth in science production. In our study, the total number of publications was 3,771 between 1993 and 2013 (average of 188 papers per year). A recent paper (3) showed the total number of urology and nephrology publications in Arab countries (21 countries) for 40 years from 1972 - 2011 as 224,479 in web of science database (Average of 267 papers per year for each country). However, these results are not comparable due to their differences in years and database. During 2000 - 2009, according to the findings of the present study, the number of articles written by Iranians was 1,777, while at the same time (in a decade), the number of articles in China and Saudi Arabia were 3,100 and 462 , respectively $(3,4)$. Some studies proposed that lack of funding may be the cause of low scientific research output in the Arab world $(5,6)$. It is also, possible that the overall growth of published articles of Iranians or some other scientific societies is partly due to increased number of foreign international conferences, which can significantly affect the rate of publication in internationally published journals. Furthermore, Nourbala and his coworkers (7) showed that Iran had the maximum number of publications in the field of transplantation after Turkey among Muslim counties in a period of eight years (2000 - 2007). This shows the high interest and experience of Iranian researchers and urologists in transplantation. We also showed that transplantation was the most prevalent topic in nephrology and urology papers ( $44.6 \%, 1682$ papers).

Some reasons for the rising trend observed in the quantity might be firstly the huge population, secondly funding in the field of nephron-urology, thirdly increased number of researchers and subspecialists in this field and finally publishing articles in domestic journals and fourthly, gov- 
ernment overall policies to promote research in Iran. Nevertheless, the number of publications derived from Medline-indexed journals cannot be a good representative of all Iranian scientific nephrology and urology population and this amount merely reflects the overall estimation.

We showed that the mean journals Impact Factor (IF) within five years decreased slightly. Figure 3 shows a steep decline from 1994 to 2008 in the mean IF for journals in which Iranian articles were published. After that a notch is seen and then a descending growth until 2013. The jumps observed within five years might be due to increasing number of Iranian international journals indexed in ISI and increase in their impact factor during these years. It is hoped that this upward trend would be continued. Despite the high level of mean impact factor in the past years, the number of articles was lower. However, the mean of impact factor is reduced, the amount of articles published in high impact factor journals are significantly increased.

Due to the wide variety of Nephrology and Urology, certainly a lot of complex issues in these areas are unknown and remain unknown and require further investigation. We found that the subject of kidney transplantation has received the most attention in the recent years and most papers topics in our study were about transplantation (44.6\%). The number of transplant centers and transplanted patients increased during the past two decades in Iran (8). Iran has been the most active country in the field of transplantation in the Middle East. The annual rate of kidney transplantation in Iran ranks the highest among the Middle Eastern countries (9). Regarding the type of articles published in journals, our results were relatively similar to previous studies to a large extent. Most of these studies were retrospective articles, while a few contributed to clinical trial studies. Zhou et al. (4) reported that based upon a ten yearanalysis in China, the most published articles in the field of nephrology and urology were case reports, randomized control trials and clinical trial articles, respectively. These results are in contrast with our study. In retrospective studies, the researcher analyzes patients' records and therefore, has lower scientific value than prospective studies. Most sources of error due to confounding factors and bias are more prevalent in retrospective studies than prospective ones (10), so, more attention should be paid in these cases. Case reports are a scientific documentation of a single clinical observation. It can add to our knowledge about the etiology, pathogenesis, natural history and treatment of rare diseases, and is so useful for training junior investigators (11). Given less time requirement to write and present this paper and easier publishing process than other types of articles in journals, many researchers are willing to publish case reports (12). However, performing a randomized controlled trial or analytical epidemiological studies requires long time, manpower and high cost (13).

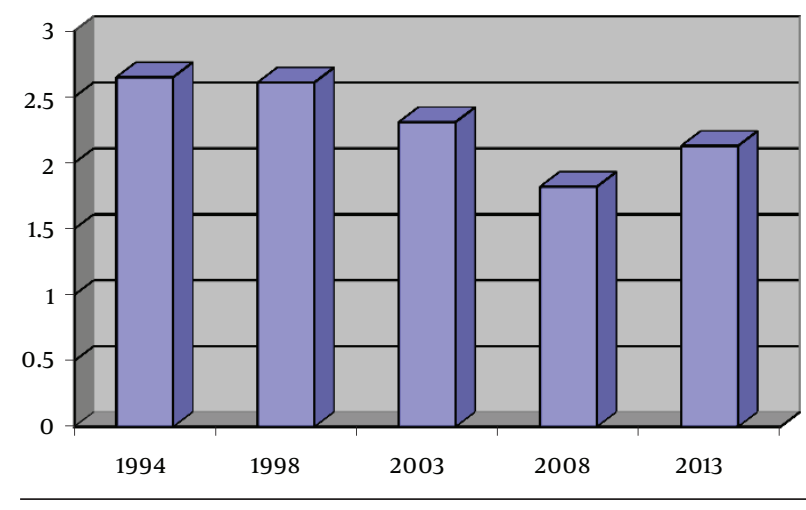

Figure 3. Mean Journals IF in Five Years

The results of this study showed that Tehran university of medical sciences has the greatest amount of participation, cooperation and contribution in Nephrology and Urology published-articles among Iranian universities and scientific institutes. A study conducted by Einollahi et al. in 2007 showed that most Iranian biomedical papers (according to the first author in the field of nephrology) were from Tehran city (55.2\%), particularly from some academic centers such as Iran university (15.0\%), Shahid Beheshti university (13.0\%), Tehran university (11.2\%) and Baqiyatallah university (10.2\%). In the present study, we examined publications of urologists and nephrologists together. It showed that these statistics have changed in 2013. Therefore, Tehran University with $18 \%$ had the highest number of articles and also the highest growth among the universities from 2007 up to now.

Iranian universities are very competitive to publish research articles and more important promote medical sciences. Some, if not the only, reasons for research activities and positive movement made in Iranian research community might be increasing encouragement system for researchers, well-development of research culture, fellowship training in Nephrology and urology and also setting up a Research PhD degree in universities.

Finally, our study had some limitations. We used PubMed center database, which is easily accessible and widely used. However, some articles are not included in this database and accordingly were not analyzed in our study. In PubMed, only the address of the first author is presented, so it may limit the researcher to understand other centers and affiliations involved in the project.

In conclusion, Iranian publications in the field of nephrology and urology have had a considerable and significant increase in the recent years in Middle Eastern countries, but there is a wide distance to be a science exporter country in the world.

\section{Acknowledgements}

We would like to thank Dr. Salesi for his helps for statistical analysis. 


\section{Authors' Contributions}

Study concept and design, acquisition of data: Mohsen Motalebi and Mehrdad Ebrahimi. Analysis and interpretation of data: Mohsen Motalebi. Drafting of the manuscript: Behzad Einollahi and Mohsen Motalebi. Critical revision of the manuscript for important intellectual content: Behzad Einollahi. Statistical analysis: Mohsen Motalebi, Mehrdad Taghipour, and Mehrdad Ebrahimi. Administrative, technical and material support: Behzad Einollahi, Mohsen Motalebi, and Mehrdad Taghipour. Study supervision: Behzad Einollahi.

\section{References}

1. Friedman DJ, Starfield B. Models of population health: their value for US public health practice, policy, and research. Am J Public Health. 2003;93(3):366-9.

2. Malekzadeh R, Mokri A, Azarmina P. Medical science and research in Iran. Arch Iran Med. 2001;4(1):27-39.

3. Sweileh WM, Zyoud SH, Al-Jabi SW, Sawalha AF. Assessing urology and nephrology research activity in Arab countries using ISI web of science bibliometric database. BMC Res Notes. 2014;7:258
4. Zhou X, Xing C, Xin L, Hu H, Li L, Fang J, et al. Scientific publications in urology and nephrology journals from China: A 10-year analysis. Can Urol Assoc J. 2012;6(2):102-6.

5. Benamer HT, Bakoush O. Arab nations lagging behind other Middle Eastern countries in biomedical research: a comparative study. BMC Med Res Methodol. 2009;9:26.

6. Bredan A, Benamer H, Bakoush O. Visibility of Arab countries in the world biomedical literature. Libyan J Med. 2011;6.

7. Nourbala MH, Taheri S, Habibi R, Abolhasani E, Nemati E, Pourfarziani V, et al. "Transplantation" research output by Muslim nations: current status, trends and future outlook. Ann Transplant. 2008;13(2):21-7.

8. Einollahi B. Nephrology research output in Iran in a decade. Iran JKidney Dis. 2007;1(2):57-62.

9. Mahdavi-Mazdeh M, Heidary Rouchi A, Norouzi S, Aghighi M, Rajolani H, Ahrabi S. Renal replacement therapy in Iran. Urol J. 2007;4(2):66-70.

10. Li J, Gao XH, Bian Q, Guo ZY, Mei XB, Yu G, et al. Comparative study of scientific publications in urology and nephrology journals originating from USA, China and Japan (2001-2010). PLoS One. 2012;7(8):e42200.

11. Carey JC. The importance of case reports in advancing scientific knowledge of rare diseases. Adv Exp Med Biol. 2010;686:77-86.

12. Vandenbroucke JP. In defense of case reports and case series. Ann Intern Med. 2001;134(4):330-4.

13. Peduzzi P, Henderson W, Hartigan P, Lavori P. Analysis of randomized controlled trials. Epidemiol Rev. 2002;24(1):26-38. 